\title{
Simulation and Correction Of Triana-Viewed Earth Radiation Budget with ERBE/ISCCP Data
}

\author{
Jianping Huang \\ AS\&M, Inc. \\ Hampton, VA USA \\ Patrick Minnis \\ NASA Langley Research Center \\ Hampton, Virginia USA \\ David R. Doelling \\ AS\&M, Inc. \\ Hampton, VA USA \\ Francisco P. J. Valero \\ Atmospheric Research Laboratory \\ Scripps Institute of Oceanography \\ La Jolla, CA USA
}

IGARSS 2002

Toronto, Canada

24-28 June 2002 


\title{
SIMULATION AND CORRECTION OF TRIANA-VIEWED EARTH RADIATION BUDGET WITH ERBE/ISCCP DATA
}

\author{
J. Huang ${ }^{1}$, P. Minnis ${ }^{2}$, D. R. Doelling ${ }^{1}$, F. P. J. Valero ${ }^{3}$ \\ ${ }^{1} \mathrm{AS} \& \mathrm{M}$, Inc. \\ Hampton, Virginia USA \\ ${ }^{2}$ Atmospheric Sciences \\ NASA Langley Research Center \\ Hampton, Virginia USA \\ ${ }^{3}$ Atmospheric Research Laboratory \\ Scripps Institute of Oceanography \\ La Jolla, CA USA
}

\begin{abstract}
This paper describes the simulation of the earth radiation budget (ERB) as viewed by Triana and the development of correction models for converting Trianaviewed radiances into a complete ERB. A full range of Triana views and global radiation fields are simulated using a combination of datasets from ERBE (Earth Radiation Budget Experiment) and ISCCP (International Satellite Cloud Climatology Project) and analyzed with a set of empirical correction factors specific to the Triana views. The results show that the accuracy of global correction factors to estimate ERB from Triana radiances is a function of the Triana position relative to the Lagrange-1 (L1) or the Sun location. Spectral analysis of the global correction factor indicates that both shortwave (SW; $0.2-5.0 \mu \mathrm{m}$ ) and longwave (LW; $5-50 \mu \mathrm{m}$ ) parameters undergo seasonal and diurnal cycles that dominate the periodic fluctuations. The diurnal cycle, especially its amplitude, is also strongly dependent on the seasonal cycle. Based on these results, models are developed to correct the radiances for unviewed areas and anisotropic emission and reflection. A preliminary assessment indicates that these correction models can be applied to Triana radiances to produce the most accurate global ERB to date.
\end{abstract}

\section{INTRODUCTION}

Triana is designed to continually monitor the sunlit side of the earth and promises to offer new insights into how our planet's climate works as an integrated system [1]. It will be the first Earth-observing satellite in a Lagrange-1 (L1) position at distance of roughly 1.5 million kilometers from the Earth. The Ll point is the location where the Earth's gravitational field equally counters that of the sun. Since the strength of the gravitational attraction determines the orbital period, Triana will orbit the sun at the same rate as the Earth.
Triana will be placed in an elliptical Lissajous orbit about L1 and will vary from $4^{\circ}$ to $15^{\circ}$ about the Earth-sun line. It will continuously measure between 92 and $97 \%$ of the sunlit Earth, never viewing the dark side of the planet. Triana's Earth-viewing instruments consist of the ScrippsEPIC (Earth Polychromatic Imaging Camera), a 10channel imager, and the Scripps-NISTAR (National Institute of Standards and Technology Absolute Radiometer), a single-pixel 4-channel broadband cavity radiometer. Data from the Scripps-EPIC and ScrippsNISTAR will be used to monitor the Earth's radiant power and to analyze weather systems and cloud patterns in an entirely new way.

ERB measurements at the top of the atmosphere (TOA) are fundamental quantities for monitoring the global climate system. These measurements have traditionally been obtained from ERB instruments on polar-orbiting satellites that typically view a region of the earth only twice each day. They cannot provide continuous spatial coverage of the Earth's entire surface at a specific time or provide continuous temporal coverage for a specific location. NISTAR is designed to facilitate determination of the radiation budget for an entire hemisphere every $10 \mathrm{~min}$ or less from a single set of 3 measurements. However, to derive the ERB from these radiances, it is necessary to convert each radiance to a flux and to account for the radiation field of the dark half of the earth. The Earth's surface and atmosphere are anisotropic reflectors and emitters resulting in a relatively complex variation of radiance leaving the Earth as a function of the viewing and illumination conditions. Triana views the Earth from a limited range of angles corresponding to scattering angles between $165^{\circ}$ and $176^{\circ}$. Therefore, to convert radiance to flux requires the use of anisotropic directional models (ADM) to account for the emittance and reflectance anisotropies. Additionally, a sliver of the sunlit Earth (missing light) is 
out of view (replaced by a dark sliver) because the satellite will not be positioned exactly on the Earth-sun line. This missing light must be taken into account for a complete ERB. Finally, no LW measurements are taken at night. Thus, some means is needed to account for the LW fluxes at night. This paper describes the simulation of a Triana-viewed ERB and the development of correction models using combinations of ERBE and ISCCP data.

\section{ERBE/ISCCP DATA}

ERBE data were collected from three satellites, the Earth Radiation Budget Satellite ERBS, NOAA-9, and NOAA-10 [2]. Each satellite measured broadband SW and LW radiances with cross-track scanners and widefield-of-view radiometers. The scanner data are used here because they provide the spatial resolution and scene type information needed to convert radiances to fluxes. Because the ERBE data are temporally sparse, interpolation techniques are used to estimate the values at each hour based on only a few samples per day. To improve on the ERBE sampling, 3-hourly geostationary data are used to fill the gaps between the ERBE measurements [3]. ISCCP DX geostationary 3-hourly visible $(0.65 \mu \mathrm{m})$ and infrared (IR, $11 \mu \mathrm{m})$ radiances [4] were gridded into the $2.5^{\circ}$ equal-angle regions used by ERBE. Narrowband-to-broadband conversion coefficients were computed for ocean, land and snow to use in standard formulae [5] for each geostationary satellite and month using coincident and collocated ERBE fluxes and ISCCP fluxes. The latter were derived from the ISCCP radiances by application of ERBE bidirectional reflectance and limb-darkening models $[6,7]$. The IR coefficients were not based on scene type. The narrowband-to-broadband conversions were then applied to all of the ISCCP radiances to compute a SW albedo and a longwave flux for each $2.5^{\circ}$ region. The ISCCPderived broadband fluxes were then normalized to the existing ERBE fluxes using the ratio of the ERBE flux to the ISCCP flux at the nearest time of coincidence for both satellites. Since polar-orbiting satellites cross the poles 14 times a day, the ISCCP broadband fluxes were only applied to regions between $60^{\circ} \mathrm{S}$ and $60^{\circ} \mathrm{N}$. The dataset then consists of 3-hourly ISCCP-based fluxes and scene information (percent cloud cover, surface type) combined with ERBE fluxes and scene types at other hours. Fluxes for the remaining hours without data are filled in using the ERBE interpolation methods to provide a complete hourly dataset for the entire globe. The inclusion of the ISCCP data provide a more realistic hourly dataset than previously used [8] because the ISCCP data measure the actual changes in cloud cover and the resulting fluxes that were only estimated when using the ERBE data alone [8]. This combination of multiple satellites is used to simulate global radiances that would be measured by Triana.

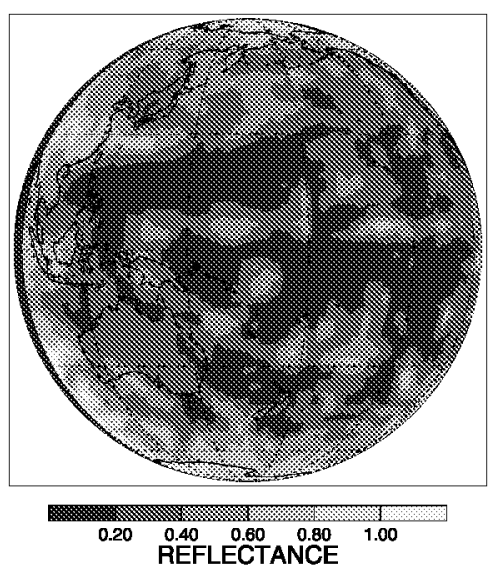

Fig. 1. Simulated Triana-viewed reflectance from ERBE data for March 21, 1986 at $15^{\circ}$ east from L1.

\section{SIMULATION OF TRIANA-VIEWED ERB}

The simulation of the Triana-viewed radiances is used to generate correction factors that can be applied to NISTAR-measured radiances to compute global SW albedo and LW flux. Having the hourly fluxes at a $2.5^{\circ}$ scale, it is possible to compute the Triana-viewed radiance at any set of viewing and illumination angles by specifying the location of the satellite in the Lissajous orbit, computing the solar and viewing zenith angles and relative azimuth angle for each viewed region, and then multiplying the SW and LW flux for the region by the bidirectional reflectance and limb-darkening factors for the scene $[6,7]$. The radiances and fluxes are then integrated over the viewed disc. The fluxes for the entire globe are also computed for the same time.

Figure 1 shows an example of Triana-viewed regional reflectance assuming an offset position of $15^{\circ}$ East from L1. The dark sliver on the left-hand side of the figure corresponds to the unilluminated portion of the Triana-viewed half disc. A corresponding sunlit sliver behind the right side of the disc out of the field of view is referred to as the missing light area. It follows that the global albedo at any given time must account for the anisotropy of the viewed $\mathrm{SW}$ radiance such that the albedo derived from a radiance for the viewed disc should match the integrated albedo from a scene like that in Fig. 1. Additionally, the contribution of the missing light area must be taken into account so that the albedo of the measured area is adjusted to match the global albedo. The first SW correction factor is the global reflectance anisotropic factor (GRAF) that is simply the ratio of the disc albedo to the disc reflectance. The missing light or albedo correction factor (ACF) is the ratio of the global albedo to the disc albedo. Thus, the global albedo for a given view then is the product of the measured reflectance, the GRAF, and the ACF for the particular viewed disc. 

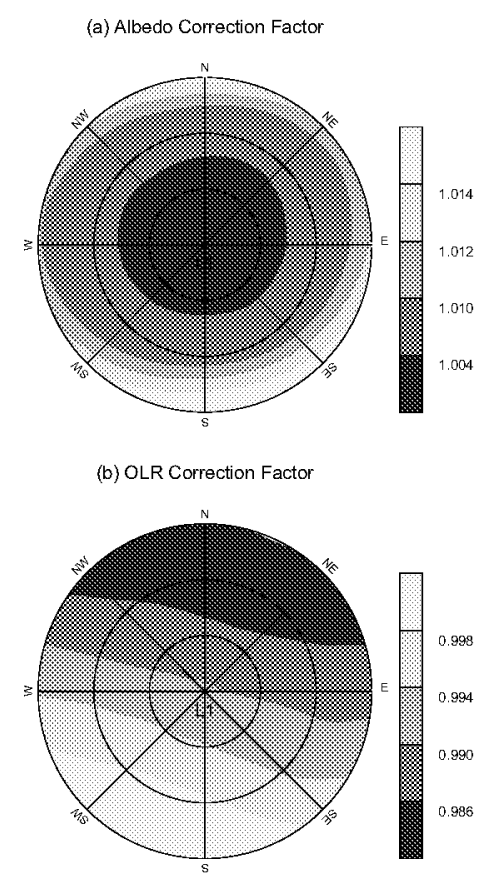

Fig.2. Monthly mean global Triana missing light albedo and nighttime correction factors as functions of LO at 0000 UTC for September 1987.

Similarly, the observed disc LW radiance must be corrected for limb darkening and for the unviewed part of the Earth at each hour to compute the global outgoing LW radiation (OLR). The global limb-darkening factor (LDF) is the ratio of the disc OLR to the disc radiance, while the nighttime OLR correction factor (OCF) is the ratio of the global OLR to the disc OLR.

Figure 2(a) shows the monthly mean values of the global ACF as a function of L1 offset position, LO, at 0000 UTC, September 1987. The values of ACF increase monotonically with distance from L1. The maximum missing light albedo factor appears near $15^{\circ} \mathrm{N}$ or $15^{\circ} \mathrm{S}$ off L1 because significant portions of the highly reflective polar regions are within the relatively large unviewed sliver of the sunlit disc. At the equinox, both poles are equally sunlit. However, observing only one pole is apparently not sufficient to compensate for the unviewed polar region. Values for the GRAF (not shown) can be as large as 1.2 as LO approaches L1. Figure 2(b) shows the mean values of OCF for the same time and month. The patterns of the nighttime OLR correction factor are very different from those for albedo (Fig. 2a), which is more symmetrical around the L1 point. The lowest values of OCF $(\sim 0.986)$, result when LO is northeast of L1 and increase gradually towards the southwest to values of $\sim 1.0$. This plot indicates that the Triana-viewed disc OLR is $1.64 \%$ greater than the global OLR at the southeast point. Values for LDF (not shown) can be as small as 0.970 . (a) $15^{\circ}$ East from $L 1$

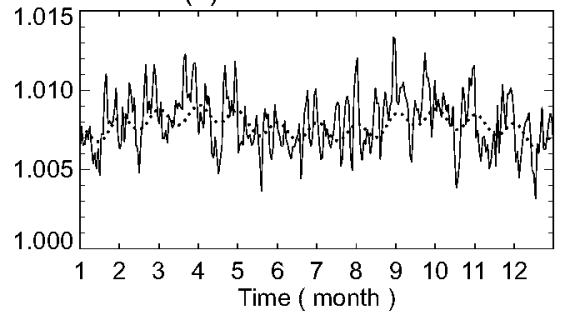

(b) $15^{\circ}$ West from $\mathrm{L} 1$

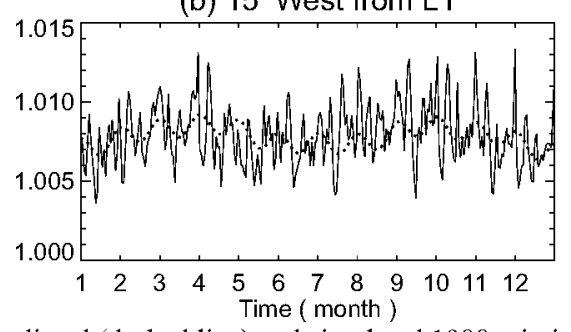

Fig. 3. Predicted (dashed line) and simulated 1988 missing light albedo correction factors (solid line) for (a) $15^{\circ}$ East from L1; (b) $15^{\circ}$ West from L1.

\section{DEVELOPMENT OF CORRECTION MODELS}

Time series analysis indicates that the long-term trend, diurnal and seasonal variations are also significant for all of the correction factors. Therefore, the correction model should include the long-term trend, the seasonal cycle, and the seasonally modulated diurnal cycle. For any time and position, the general correction model can be written as:

$$
\begin{gathered}
Y\left(T_{d}, T_{g}, \phi, \theta\right)=C_{0}(\phi, \theta)+C_{I}(\phi, \theta)^{*} T_{d}+C_{2}(\phi, \theta)^{*} T_{d}^{2}+ \\
C_{3}(\phi, \theta)^{*} \cos \left(2 \pi f_{d} T_{d}\right)+C_{4}(\phi, \theta)^{*} \cos \left(2 \pi f_{g} T_{g}\right)+ \\
C_{5}(\phi, \theta)^{*} \cos \left(2 \pi f_{d} T_{d}\right)^{*} \cos \left(2 \pi f_{g} T_{g}\right)
\end{gathered}
$$

where $\phi$ is the angle from North (in degrees), $\theta$ is the distance from L1 (in degrees), and $f_{d}$ and $f_{g}$ are the frequencies of the seasonal and diurnal cycles, respectively. The last term in equation (1) represents the effect of modulation. The coefficients, $\left(C_{i}(\phi, \theta), \mathrm{I}=0, \ldots 5\right)$ were computed using a least squares multiple regression fit for each position. These models can be used to predict the hourly correction factors for any given time $\left(T_{d}, T_{g}\right)$ and position $(\phi, \theta)$. Three years (Jan. 1985 to Dec. 1987) of simulated data are used as the historical dataset to determine the regression coefficients. The resulting coefficients were used to predict the factors for 1988 and then compared with the simulated data. Examples of the predicted missing light albedo correction factors at 2 maximum LOs are shown in Fig. 3. The predicted (dashed line) and simulated (solid line) albedo correction factor values are in good agreement. The respective mean and standard deviation of the differences are 0.00021 and 0.0017 . Preliminary results indicate that the correction model is capable of resolving the global correction factor. 


\section{CONCLUSIONS AND DISCUSSIONS}

This paper provides a basic framework for the simulation of Triana-viewed ERB. The preliminary prediction results indicate that these correction models can be used to produce the most accurate global ERB to date. However, the correction models are purely statistical and it is not possible to distinguish between physical and random relationships in the data. Also, the models may be biased by the sampling patterns of the ERBE satellites. Correction factors based on the combined ERBE and ISCCP datasets provide a more accurate representation of the diurnal cycle. A more advanced approach using cloud information from the EPIC is also under development to explicitly account for physical variations in each viewed scene that are not taken into account with the purely statistical method used here. In the meantime, the technique developed here should provide a highly reliable method for monitoring the global radiation balance from Triana.

\section{REFERENCES}

[1] F. P. J. Valero, J. Herman, P. Minnis, W. D. Collins, R. Sadourny, W. Wiscombe, D. Lubin, and K. Ogilvie: "Triana - a Deep Space Earth and Solar Observatory," NASA background report, 1999.

[2] B. R. Barkstrom, E. F. Harrison, G. L. Smith, "Results from the Earth Radiation Budget Experiment (ERBE)," Adv. Space Res., 9, pp. 775 - 782, 1989.

[3] D. F. Young, P. Minnis, D. R. Doelling, G. G. Gibson and T. Wong: "Temporal interpolation methods for the Clouds and the Earth's Radiant Energy System (CERES) Experiment," J Appl. Meteorol, 37, pp. 572$590,1998$.

[4] W. B. Rossow and R. A. Schiffer, "Advances in understanding clouds from ISCCP," Bull. Am. Meteorol. Soc., 80, pp. 2261-2287, 1999.

[5] P. Minnis and W. L. Smith, Jr.: "Cloud and radiative fields derived from GOES-8 during SUCCESS and the ARM-UAV Spring 1996 Flight Series," Geophys. Res. Lett., 25, pp. 1113-1116, 1998.

[6] J. T. Suttles, R. N. Green, et. al.: “Angular radiation models for Earth-atmosphere system: Volume 1 Shortwave radiation," NASA RP 1184, Vol. I, 1988.

[7] J.T. Suttles, R. N. Green, et al., "Angular radiation models for the Earth-atmosphere systems: Volume II: Longwave radiation," NASA RP 1184, Vol. II, 1989.

[8] P. Minnis, J. Huang, D. R. Doelling, and F. P. J. Valero: "Simulation and correction of Triana-viewed Earth radiation budget with ERBE data," Proc. SPIE Conf. on Sensors, Systems, and Next-Generation Satellites V, Toulouse, France, Sept. 12-17, pp. 391$401,2001$. 